\title{
El enojo como rasgo de personalidad y agresión física en adolescentes de preparatoria
}

\section{Anger as personality trait and physical aggression in high school adolescents}

Raúl J. Alcázar-Olán

Universidad Iberoamericana Puebla

\author{
Samuel Jurado Cárdenas \\ Verónica Reyes Pérez \\ Universidad Nacional Autónoma de México
}

\begin{abstract}
Resumen
La presente investigación comparó a jóvenes de preparatoria $(M$ de edad $=15.92, D E=0.9)$ con alto y bajo enojo rasgo con respecto a cuándo, dónde y hacia quién mostraron su enojo por medios físicoagresivos (e. g., golpear a alguien, dañar objetos). Los grupos de alto y bajo enojo rasgo quedaron definidos por la pertenencia de sus integrantes al cuartil superior (alto enojo, $n=125$ ) e inferior (bajo enojo, $n=149$ ) en la Escala de Enojo Rasgo (Moscoso \& Spielberger, 1999). Dicha escala midió el patrón o tendencia de los adolescentes a molestarse en cualquier lugar y con cualquier persona. Como resultado, a diferencia de los jóvenes con bajo enojo, los de alto enojo: 1) mostraron su molestia con agresiones físicas, recientemente, hacia otras personas (e. g., empujar a alguien) y el ambiente (e. g., golpear la pared); 2) aventaron cosas a sus hermanos/as durante el último mes, la última semana e incluso hoy; y 3 ) azotaron la puerta estando enojados en la mañana, en la tarde y en la noche, cuando estuvieron en casa. Los datos indican que las relaciones de los adolescentes con personas cercanas (hermanos/as) y sus propias casas fueron el contexto donde los episodios de enojo con agresiones físicas surgieron en mayor medida, especialmente en los jóvenes con alto enojo rasgo.

Palabras clave: adolescencia, agresión, enojo, enojo rasgo, preparatoria.
\end{abstract}

Nota del autor

Raúl J. Alcázar-Olán, Departamento de Ciencias de la Salud, Universidad Iberoamericana Puebla (UIP); Samuel Jurado Cárdenas, Facultad de Psicología, Universidad Nacional Autónoma de México (UNAM); Verónica Reyes Pérez, Facultad de Psicología, UNAM.

La correspondencia en relación con este artículo debe dirigirse a Raúl J. Alcázar-Olán, Departamento de Ciencias de la Salud, UIP, bulevar del Niño Poblano, número 2901, colonia UT-Atlixcáyotl, C. P. 72197, Puebla, Pue., México.

Dirección electrónica: raul.alcazar@iberopuebla.mx, rulet7@gmail.com 


\begin{abstract}
This study compared high and low trait anger youth $(M$ of age $=15.92, S D=0.9)$ from high school in terms of when, where and towards whom they expressed their anger in physically aggressive ways (e. g., hitting someone, hitting objects). High and low trait anger youth were those who scored in the upper (high anger, $n=125$ ) and lower (low anger, $n=149$ ) quartile in the Trait Anger Scale (Moscoso $\&$ Spielberger, 1999). This scale measured the anger proneness or the tendency to get angry in any place with any person. As a result, compared to low anger adolescents, those with high trait anger: 1) showed their anger with physical aggression recently toward other people (e. g., pushing someone) and toward the environment (e. g., hitting the wall); 2) throwed things to their brothers and sisters during the last month, the last week and even today: and 3) slammed their home doors throughout the day (morning, afternoon, and night). The results indicate that relationships with close persons (brothers, sisters) and the adolescents' houses are the contexts where anger episodes emerged in greater extent, particularly in adolescents with high trait anger.

Keywords: adolescence, aggression, anger, trait anger, high school.
\end{abstract}

La comparación entre a jóvenes con alto y bajo enojo rasgo en cuanto a sus agresiones físicas (e. g., golpear, empujar a alguien) fue el primer motivo de la presente investigación. La adolescencia es una etapa crucial donde algunos jóvenes presentan más episodios de enojo y menor control de la emoción que los adultos (Alcázar-Olán \& Deffenbacher, 2013a; Blanchard-Fields \& Coats, 2008; Hubbard, Romano, McAuliffe, \& Morrow, 2010). El enojo en jóvenes debe estudiarse porque se asocia con agresión (Campano \& Munakata, 2004), ideas de suicidio (Lee, Choi, Kim, Park, \& Shin, 2009), depresión, uso de alcohol y drogas (Cautin, Overholser, \& Goetz, 2001). Una forma problemática del enojo es cuando incluye el deseo de hacer daño. El manejo inadecuado del enojo lleva a conductas agresivas y daños físicos contra la persona enojada, aquellos a su alrededor y su ambiente (Loeber \& Hay, 1997; Peled \& Moretti, 2007).
El enojo como rasgo de personalidad es un patrón relativamente estable de pensar, sentir y actuar en cuanto al grado en que los adolescentes son propensos a sentir ira en cualquier lugar y contra cualquier persona (Alcázar-Olán \& Deffenbacher, 2013a). El enojo rasgo es la característica que marca la diferencia entre unos individuos y otros en términos de mayor irritabilidad y enojo (Alcázar-Olán \& Deffenbacher, 2013b; Spielberger, 1999).

Los jóvenes con alto enojo rasgo son muy iracundos y reactivos ante cualquier situación frustrante (Alcázar-Olán \& Deffenbacher, 2013b; Quinn, Rollock, \& Vrana, 2014). Discuten, son agresivos, guardan rencores y el enojo les dura mucho tiempo; es fácil que sientan que los demás los están provocando. Por el contrario, los jóvenes con bajo enojo rasgo conservan, generalmente, la calma y controlan sus reacciones verbales y conductuales; piensan antes de actuar, sus enojos son menos intensos, menos frecuentes y de corta duración. 
Las diferencias entre las personas de alto y bajo enojo están descritas en la teoría del enojo estado-rasgo (Alcázar-Olán \& Deffenbacher, 2013b; Spielberger, 1999; Deffenbacher et al., 1996). La teoría predice que a diferencia de los individuos con bajo enojo rasgo, los de alto enojo rasgo son más agresivos (hipótesis de agresión), se molestan por más situaciones (hipótesis de provocación), manejan su enojo de forma disfuncional (hipótesis de enfrentamiento positivo reducido), se molestan de forma intensa (hipótesis de intensidad) y frecuente (hipótesis de frecuencia), mantienen su enojo por mucho tiempo (hipótesis de duración) y experimentan consecuencias más severas por su enojo (hipótesis de consecuencias negativas). Como resultado, las personas con alto enojo rasgo tienen dificultades para controlar sus conductas y sus emociones (i.e., difícilmente reducen su enojo).

Los planteamientos de la teoría del enojo estado-rasgo han sido comprobadas constantemente en adultos (Deffenbacher et al., 1996) y en adolescentes (Alcázar-Olán \& Deffenbacher, 2013a; Quinn et al., 2014), lo cual indica solidez de la teoría, pues se presentan claras diferencias entre individuos de alto y bajo enojo rasgo.

Comparados con personas de bajo enojo rasgo, las agresiones de jóvenes con alto enojo rasgo son más frecuentes, por ejemplo, rompen cosas, avientan objetos, empujan y golpean a otros individuos (Alcázar-Olán \& Deffenbacher, 2013a); sin embargo, las investigaciones no han cuantificado hasta ahora el término "frecuente": ¿frecuente significa una vez al año?, ¿una vez al mes?, ¿una vez al día? (Schwarz, 1999; Winkielman, Knäuper, \& Schwarz, 1998). Si una per- sona con alto enojo rasgo expresa su agresión frecuentemente, ¿cuándo fue la última vez que lo hizo?, es decir, ¿cuándo fue la última vez que aventó cosas o empujó a alguien? No sabemos de una investigación que se haya enfocado en estas preguntas. Las respuestas servirían para identificar algunas características de las expresiones del enojo por medios físico-agresivos en jóvenes con alto enojo rasgo. Para responder algunos de estos planteamientos, comparamos a jóvenes con alto y bajo enojo rasgo para obtener un panorama sobre cuándo, dónde y con quién expresaron su enojo con agresión física.

El objetivo de investigación fue caracterizar las expresiones agresivas de los adolescentes cuando están molestos. En consecuencia, comparamos a jóvenes con alto y bajo enojo rasgo para responder las siguientes preguntas: 1) ¿quiénes mostraron conductas agresivas más recientemente, los jóvenes con alto o bajo enojo rasgo?, 2) si jóvenes expresaron su enojo por medio de conductas agresivas hacia otra persona, ¿cuándo fue la última vez que lo hicieron y quién recibió la agresión?, 3) una vez que los jóvenes expresaron su enojo por medio de conductas agresivas hacia el ambiente (e. g., golpear la pared), ¿cuándo fue la última vez que lo hicieron, en qué momento (mañana, tarde o noche) y en qué lugar (casa o escuela)? Las respuestas a estas preguntas permitirán una descripción más amplia de las expresiones agresivas en jóvenes con alto y bajo enojo rasgo.

Contrario a lo esperado, la mayoría de las investigaciones, incluyendo estudios metaanalíticos (Archer, 2004), indica que varones y mujeres son muy similares cuando están enoja- 
dos; no obstante, incluimos la variable sexo para examinar si influye en los resultados.

\section{Método}

\section{Participantes}

La muestra inicial fue de 522 adolescentes de una preparatoria pública matutina de la ciudad de Puebla, México. Se eliminaron 12 casos debido a que respondieron incorrectamente el cuestionario (i. e., marcaron varias respuestas cuando debían elegir sólo una). La media de edad fue de $15.92(D E=0.90) ; 43 \%$ cursaba el primer año; $37 \%$, segundo; y $20 \%$, tercero. El $6.1 \%$ era hijo único y los demás tenían hermanos o hermanas. El 84 \% dijo ser católico; el $8 \%$, no tener una religión; y $8 \%$ informó estar en una religión no católica.

En apego al objetivo de investigación se formaron dos grupos, uno de alto y otro de bajo enojo rasgo. Los grupos de alto y bajo enojo quedaron definidos por la pertenencia de los adolescentes al cuartil superior (alto enojo, puntajes mayores a 23) e inferior (bajo enojo, puntajes menores a 16) en la Escala de Enojo Rasgo (EER). En consecuencia, el grupo de alto enojo tuvo 125 participantes (36 hombres y 89 mujeres) y el de bajo enojo 149 (68 varones y 81 mujeres).

\section{Instrumentos}

EER. La escala tiene diez reactivos sobre la propensión o tendencia a molestarse a través del tiempo y las situaciones (Moscoso \& Spielberger, 1999). Mide el grado en que una persona es frecuentemente enojona. Los datos de validez en jóvenes mexicanos (Alcázar-Olán,
Deffenbacher, Pool-Cibrián, Reyes-Pérez, \& Hernández-Guzmán, 2014) indican que el enojo rasgo tiene dos subescalas, igual que la escala original (Moscoso \& Spielberger, 1999; Spielberger, 1999). Una subescala mide la reacción de enojo ante situaciones frustrantes $(5$ reactivos, $\alpha=.78$-.79, e. g., me pongo furioso cuando me critican delante de los demás) y la otra mide el temperamento enojón ( 5 reactivos, $\alpha=.79-.85$, e. g, soy muy irritable, me enojo fácilmente), que se refiere a la tendencia a reaccionar con enojo ante la menor provocación. Las opciones de respuesta son "Casi nunca" (valor $=1$ ), "A veces" (valor $=2)$, "Frecuentemente" (valor $=3$ ) y "Casi siempre" (valor $=4$ ). Mayores puntajes indican mayor enojo rasgo.

Cuestionario de Expresión del Enojo con Agresión Física. El cuestionario se construyó para el presente estudio en apego a los objetivos y consta de dos subescalas: seis reactivos de Expresión del enojo con agresión física hacia las personas (e. g., retar a los golpes) y seis reactivos de Expresión del enojo con agresión física hacia el ambiente (e. g., azotar la puerta). Los adolescentes respondieron a la pregunta “¿Cuándo fue la última vez que estando enojado..." y luego aparecían los reactivos. Las opciones de respuesta oscilaron desde "Lo hice hoy" (valor $=7)$ hasta "Nunca lo he hecho" (valor $=1)$. Mayor puntaje indica expresión del enojo con agresión física de forma reciente.

Antes de usar el instrumento examinamos sus características de confiabilidad y validez. El cuestionario mostró validez de constructo de acuerdo con los resultados de un análisis factorial exploratorio (ejes principales y rotación 
promax) en la muestra completa $(n=510)$, el cual arrojó dos factores que explicaron $46 \%$ de la varianza. El primer factor explicó $35.21 \%$ (valor Eigen $=4.22)$ y se formó de seis reactivos que indicaban la expresión del enojo hacia otras personas (golpear, jalonear, hacer como si fuera a dar un golpe, retar a golpes, romper cosas de alguien y aventar cosas contra alguien). La confiabilidad Alfa de Cronbach fue de .77. El segundo factor explicó $10.84 \%$ de la varianza $($ valor Eigen $=1.3)$ y se formó de seis reactivos sobre expresar el enojo físicamente hacia el ambiente (dañar cosas propias, hacer como si fuera a romper algo, golpear la pared, azotar la puerta, aventar cosas y romper objetos). La confiabilidad Alfa de Cronbach fue de .71. Las dos escalas se correlacionan con moderadamente $(r=.55, p<.001)$, lo cual indica que miden un mismo constructo (agresión) en distintos aspectos (hacia las personas o hacia el ambiente). En síntesis, la escala tuvo validez de constructo y confiabilidad aceptable.

Después de los reactivos sobre agresión hacia otras personas, aparecía la pregunta sobre quiénes recibieron la agresión (el hermano, mamá, papá, compañero de la escuela, etc.). Después de los reactivos sobre agresión al ambiente (e. g., golpear la pared), aparecían las preguntas sobre cuándo lo hicieron (mañana, tarde o noche) y en dónde (casa o escuela).

\section{Procedimiento}

Pedimos autorización a los directivos de la escuela para aplicar los cuestionarios. Ellos a su vez gestionaron el permiso con el comité de padres de familia, quien también aceptó. La aplicación se llevó a cabo en los salones de clase, en ausencia del profesor. Cada grupo tuvo aproximadamente 25 alumnos. Las instrucciones se leyeron en voz alta para todo el grupo, incluyendo la aclaración del anonimato, voluntad y abstención en cuanto a responder el cuestionario. Luego se les pidió contestar en silencio; al entregar el cuestionario se agradeció su participación.

Las respuestas se capturaron en el programa SPSS versión 17 y los datos se examinaron con un análisis multivariado de varianza (MANOVA) de 2 (grupo de alto y bajo enojo rasgo) x 2 (sexo), usado por el estadístico Lambda de Wilks $(\lambda)$. Los efectos multivariados se exploraron con ANOVAs univariadas. La magnitud del efecto (Eta cuadrada, $\eta^{2}$ ) se interpretó con los criterios de Cohen (1988), donde valores de .01 a .04 se consideran pequeños, de .04 a .14 son moderados y mayores a .14 se consideran grandes. En algunos casos se compararon frecuencias por medio de ji cuadrada; sólo se informan las que fueron significativas.

\section{Resultados}

\section{Análisis preliminares}

El MANOVA mostró efectos significativos para el grupo y el sexo, Lambda de Wilks $=.749$ y $.838, F(12,258)=7.21$ y $4.14, p<.001$, Eta cuadrada $=.251$ y .162 , respectivamente; pero no para la interacción, $F(12,258)=1.33$. El tamaño del efecto multivariado fue grande para el grupo y el sexo. Ante la ausencia de interacción, las tablas muestran los efectos principales por grupo (alto y bajo enojo rasgo), y considera a varones y mujeres en conjunto. 
Los ANOVA mostraron diferencias significativas por sexo en cuatro de 12 variables. Los varones tuvieron mayores puntajes en las expresiones físico-agresivas de retar a golpes $(M$ $=2.23$ y 1.56$)$ y golpear la pared $(M=3.52 \mathrm{y}$ $2.62), F(1,272)=6.43$ у $10.26, p=.012$ у .002 , Eta cuadrada $=.023$ y .037 , respectivamente; aunque las mujeres tuvieron mayores puntajes que los varones en cuanto a aventar cosas $(M$ $=2.84$ y 2.40$)$ y azotar la puerta $(M=4.08 \mathrm{y}$ $3.33), F(1,272)=4.60$ y $9.41, p=.033$ y .002 , Eta cuadrada $=.017$ y .034 . La magnitud de los efectos fue pequeña, lo cual sugiere que el sexo explica poco, entre 1.7 y $3.7 \%$ de las diferencias en las expresiones del enojo físico-agresivas.

\section{¿Quiénes mostraron episodios agresivos recientes?}

En apego a la primera pregunta de investigación, examinamos quiénes tuvieron episodios más recientes con expresión físicoagresiva del enojo. Los grupos de alto y bajo enojo rasgo difirieron de forma consistente en todas las variables (ver tabla 1). Al compararlo con el grupo de bajo enojo rasgo, el de alto enojo tuvo conductas agresivas recientes hacia otras personas (e. g., aventar cosas contra alguien) y contra el ambiente (e. g., romper objetos). Esto indica que los adolescentes con alto enojo rasgo recurrieron últimamente a expresar su enojo de manera físico-agresiva. El tamaño del efecto varió desde pequeño hasta grande; en diez de doce variables fue moderado.

Tabla 1

Expresiones fisico-agresivas de adolescentes con alto y bajo enojo rasgo

\begin{tabular}{|c|c|c|c|c|c|c|}
\hline \multirow[t]{2}{*}{ Estando enojado... } & \multicolumn{2}{|c|}{ Bajo enojo } & \multicolumn{2}{|c|}{ Alto enojo } & \multirow{2}{*}{$\begin{array}{l}\text { Anova } \\
F \\
(1,272)\end{array}$} & \multirow[t]{2}{*}{$\eta^{2}$} \\
\hline & $M$ & $D E$ & $M$ & $D E$ & & \\
\hline 1. Aventaste cosas contra alguien & 2.49 & 1.91 & 4.03 & 2.07 & $34.01^{* *}$ & .112 \\
\hline 2. Dañaste o rompiste cosas de alguien & 1.76 & 1.45 & 2.53 & 1.77 & $13.34^{* *}$ & .047 \\
\hline 3. Golpeaste a alguien & 1.65 & 1.37 & 2.53 & 1.96 & $12.80^{* *}$ & .045 \\
\hline 4. Retaste a alguien a los golpes & 1.48 & 1.12 & 2.07 & 1.80 & $14.04^{* *}$ & .050 \\
\hline 5. Jaloneaste a alguien & 1.99 & 1.67 & 3.01 & 2.11 & $14.67^{* *}$ & .052 \\
\hline 6. Hiciste como si fueras a golpear a alguien & 2.16 & 1.88 & 2.97 & 2.12 & $11.73^{*}$ & .042 \\
\hline 7. Aventaste cosas sin importar dónde golpean & 1.77 & 1.38 & 3.51 & 1.97 & $51.08^{* *}$ & .160 \\
\hline 8. Azotaste la puerta & 3.03 & 1.81 & 4.57 & 1.74 & $36.40^{* *}$ & .119 \\
\hline 9. Dañaste o rompiste tus propias cosas & 1.52 & 1.08 & 2.68 & 2.01 & $27.17^{* *}$ & .092 \\
\hline 10. Hiciste como si fueras a golpear algún objeto & 2.05 & 1.78 & 3.07 & 2.13 & $15.96^{* *}$ & .056 \\
\hline 11. Golpeaste la pared & 2.58 & 2.04 & 3.33 & 2.10 & $9.03^{*}$ & .032 \\
\hline 12. Rompiste cualquier objeto & 1.21 & 0.84 & 1.89 & 1.63 & $14.15^{\star \star}$ & .050 \\
\hline
\end{tabular}

${ }^{*} p<.01,{ }^{* *} p<.001$.

Nota: Los reactivos 1 al 6 miden agresión física contra otras personas; del 7 al 12 la agresión física contra el ambiente. $\eta^{2}=$ Eta cuadrada (tamaño del efecto o varianza explicada). 
¿Cuándo los jóvenes agredieron y quién recibió la agresión?

La segunda pregunta de investigación se enfocó en conocer cuándo fue la última vez que el sujeto se emitió el comportamiento agresivo y quiénes recibieron las agresiones. Para ello, seleccionamos el reactivo Aventar cosas contra alguien porque tuvo el mayor puntaje en la tabla 1 , lo cual significa que dicha conducta fue la más usada recientemente para expresar el enojo de forma físico-agresiva.

El $80 \%$ de los adolescentes con alto enojo rasgo expresó su molestia por medio de aventar cosas contra alguien, mientras que $50 \%$ de los adolescentes con bajo enojo rasgo se expresó así: $X^{2}(1)=6.92, p<.01$. Al observar las situaciones recientes, donde los adolescentes tuvieron episodios físico-agresivos contra otra persona (ver tabla 2), fueron más los jóvenes con alto enojo rasgo (no los de bajo enojo rasgo) quienes aventaron cosas en los últimos días y en los últimos meses. Por ejemplo, $30 \%$ de los adolescentes con alto enojo rasgo aventó cosas contra alguien en la última semana, mientras que dicha conducta la presentó $14 \%$ de los adolescentes con bajo enojo rasgo en el mismo período: $X^{2}$ $(1)=6.42, p<.01$.

Tabla 2

Ocasión en que adolescentes de alto y bajo enojo rasgo aventaron cosas contra alguien estando molestos

\begin{tabular}{lcrcr}
\hline Cuándo lo hizo & \multicolumn{2}{c}{ Bajo enojo } & \multicolumn{2}{c}{ Alto enojo } \\
\hline & $n$ & $\%$ & $n$ & $\%$ \\
Lo hizo hoy & 1 & 0.7 & 8 & 6.4 \\
En los últimos siete días & 21 & 14.1 & 38 & 30.4 \\
En el último mes & 10 & 6.7 & 17 & 13.6 \\
En los últimos seis meses & 11 & 7.4 & 14 & 11.2 \\
En el último año & 6 & 4.0 & 8 & 6.4 \\
Hace más de un año & 25 & 16.8 & 15 & 12.0 \\
Nunca lo ha hecho & 75 & 50.3 & 25 & 20.0 \\
Total & 149 & 100 & 125 & 100 \\
\hline
\end{tabular}

Las víctimas de las agresiones fueron los hermanos y las hermanas (ver tabla 3). Los hermanos varones fueron las principales víctimas, pues recibieron agresiones con mayor frecuen- cia; $32 \%$ de los adolescentes con alto enojo rasgo aventó cosas contra su hermano y $12 \%$ de los adolescentes con bajo enojo rasgo empleó dicha conducta: $X^{2}(1)=8.02, p<.01$. 
Tabla 3

Personas contra quienes los adolescentes de alto y bajo enojo rasgo aventaron cosas estando molestos

\begin{tabular}{|c|c|c|c|c|}
\hline \multirow[t]{2}{*}{ Persona a quien le aventaron cosas... } & \multicolumn{2}{|c|}{ Bajo enojo } & \multicolumn{2}{|c|}{ Alto enojo } \\
\hline & $n$ & $\%$ & $n$ & $\%$ \\
\hline Hermano & 18 & 12.1 & 40 & 32.0 \\
\hline Hermana & 12 & 8.1 & 17 & 13.6 \\
\hline Novio/a & 4 & 2.7 & 10 & 8.0 \\
\hline Compañero/a de escuela & 12 & 8.1 & 10 & 8.0 \\
\hline Mamá & 5 & 3.4 & 10 & 8.0 \\
\hline Papá & 4 & 2.7 & 3 & 2.4 \\
\hline Amiga & 5 & 3.4 & 3 & 2.4 \\
\hline Amigo & 8 & 5.4 & 2 & 1.6 \\
\hline Otro & 6 & 4.0 & 3 & 2.4 \\
\hline Nunca lo ha hecho & 75 & 50.3 & 27 & 21.6 \\
\hline Total & 149 & 100 & 125 & 100 \\
\hline
\end{tabular}

¿Cuándo y dónde los jóvenes agredieron el ambiente?

La tercera pregunta de investigación se enfocó en conocer tres puntos: la última vez, el momento (mañana, tarde o noche) y el lugar (casa o escuela) donde los adolescentes de alto y bajo enojo rasgo expresaron su enojo contra el ambiente. Usamos el reactivo de Azotar la puerta debido a que dicho comportamiento tuvo el puntaje más alto (ver tabla 1), lo cual indica uso reciente de esta conducta para mostrar el enojo de manera físico-agresiva.

El $90 \%$ de los adolescentes con alto enojo rasgo azotó la puerta alguna vez, mientras que $68 \%$ de los adolescentes con bajo enojo rasgo empleó dicha conducta (ver tabla 4). A diferencia del número de jóvenes con bajo enojo rasgo, fueron más los jóvenes con alto enojo rasgo quienes azotaron la puerta en los últimos siete días (9\% vs $30 \%$ ) y en el último mes (6\% vs
$24 \%), X^{2}(1)=11.3$ y 10.8 , respectivamente, $p<.01$. Estos datos de agresión contra el ambiente (ver tabla 4) resultaron congruentes con la información previa sobre agresión contra otras personas (ver tabla 2), lo cual indica que los jóvenes con alto enojo rasgo mostraron comportamientos agresivos recientemente como en mayor cantidad.

En cuanto al momento en que los jóvenes azotaron la puerta, tanto las personas con bajo como las de alto enojo rasgo lo hicieron con la misma frecuencia en las tardes (ver tabla 5), sin embargo, a diferencia del número de jóvenes con bajo enojo, fueron más los jóvenes con alto enojo quienes azotaron la puerta también durante las mañanas y noches. En congruencia con este resultado, el lugar donde los jóvenes con alto enojo rasgo azotaron la puerta fue, principalmente, en la casa (ver tabla 5). 
Tabla 4

Ocasión en que adolescentes de alto y bajo enojo rasgo azotaron la puerta (agresión al ambiente) estando molestos

\begin{tabular}{lrrrr}
\hline Cuándo azotó la puerta & \multicolumn{2}{c}{ Bajo enojo } & \multicolumn{2}{c}{ Alto enojo } \\
& \multicolumn{1}{c}{$n$} & $\%$ & $n$ & $\%$ \\
\hline Lo hizo hoy & 2 & 1.3 & 9 & 7.2 \\
En los últimos siete días & 13 & 8.7 & 38 & 30.4 \\
En el último mes & 9 & 6.0 & 30 & 24.0 \\
En los últimos seis meses & 25 & 16.8 & 19 & 15.2 \\
En el último año & 9 & 6.0 & 8 & 6.4 \\
Hace más de un año & 25 & 16.8 & 9 & 7.2 \\
Nunca lo ha hecho & 47 & 31.5 & 12 & 9.6 \\
Total & 149 & 100 & 125 & 100 \\
\hline
\end{tabular}

Tabla 5

Momento y lugar en que los adolescentes de alto y bajo enojo rasgo azotaron la puerta estando molestos

\begin{tabular}{lcrrr}
\hline Cuándo azotaron la puerta & \multicolumn{2}{c}{ Bajo enojo } & \multicolumn{2}{c}{ Alto enojo } \\
\hline & $n$ & $\%$ & $n$ & $\%$ \\
Mañana & 13 & 8.7 & 25 & 20.0 \\
Tarde & 65 & 43.7 & 56 & 44.8 \\
Noche & 17 & 11.4 & 27 & 21.6 \\
Nunca lo ha hecho & 54 & 36.2 & 17 & 13.6 \\
Total & 149 & 100 & 125 & 100 \\
Lugar en que azotaron la puerta & & & \\
Casa & 92 & 61.7 & 104 & 83.2 \\
Escuela & 7 & 4.7 & 4 & 3.2 \\
Otro & 1 & 0.7 & 3 & 2.4 \\
Nunca lo ha hecho & 49 & 32.9 & 14 & 11.2 \\
Total & 149 & 100 & 125 & 100 \\
\hline
\end{tabular}

\section{Discusión}

La presente investigación realizó una comparación entre jóvenes con alto y bajo enojo rasgo en cuanto a dónde, cuándo y con quién expresaron su enojo de forma físico-agresiva. Los jóvenes con alto enojo rasgo tuvieron episodios agresivos más recientes hacia otras personas (e. g., jalonear a alguien) y hacia el ambiente (e. g., romper cosas). A diferencia del número de personas con bajo enojo, fueron más los jóvenes con alto enojo rasgo quienes aventaron cosas contra alguien en el último mes ( $7 \%$ vs $17 \%$ ), 
en la última semana ( $14 \%$ vs $38 \%$ ) e incluso hoy ( $1 \%$ vs $8 \%$, respectivamente). Este resultado fue congruente con otra investigación (Alcázar-Olán \& Deffenbacher, 2013a), donde los jóvenes con alto enojo rasgo fueron más agresivos contra otras personas y contra su ambiente en comparación con que los jóvenes con bajo enojo. Esta investigación aporta al conocimiento porque indica exactamente cuándo los jóvenes mostraron su enojo con agresión. Otra aportación es la siguiente: a diferencia de los jóvenes con bajo enojo rasgo, son más los jóvenes con alto enojo quienes tuvieron episodios recientes de agresiones.

Los jóvenes aventaron cosas contra sus hermanos y hermanas, quienes fueron el principal receptor de la agresión. El resultado fue congruente con otro estudio (Averill, 1983; Schieman, 2010), en el cual los episodios de enojo sucedieron en gran medida contra personas cercanas o queridas. La aportación indica que fueron más los jóvenes de alto enojo (57\%) quienes agredieron a sus hermanos/as, mientras que pocos jóvenes con bajo enojo (20 $\%)$ cometieron estas agresiones. Las agresiones físicas contra los hermanos y las hermanas reflejan el alto enojo rasgo de los jóvenes.

Además, los jóvenes azotaron la puerta principalmente en casa, no en la escuela. Fueron más los jóvenes con alto enojo rasgo (83 \%), quienes azotaron la puerta en casa que el número de jóvenes con bajo enojo (62 \%). Si suponemos que los hermanos viven en el mismo domicilio, entonces aventarles cosas y azotar la puerta en casa ubica a los hogares como escenario donde se expresa el enojo con agresiones físicas, en jóvenes con alto enojo rasgo.

Tanto los jóvenes con alto como los de bajo enojo rasgo azotaron la puerta en las tardes. El número de personas con alto enojo rasgo que azotó la puerta en la mañana (20\% vs $9 \%$ ) y en la noche (22\% vs $11 \%)$ superó al número de jóvenes con bajo enojo. Como los jóvenes iban a la escuela en el turno matutino, entonces la conducta de azotar la puerta sucedió temprano, en casa antes de ir a la escuela. Además, como los jóvenes azotaron la puerta también en las noches, la expresión del enojo en forma físico-agresiva sucede en casa (Jackson, Kuppens, Sheeber, \& Allen, 2011). En consecuencia, los hogares de los jóvenes, más que la escuela, son el contexto donde predomina la expresión del enojo con agresión física.

Los resultados de investigación apoyan las predicciones de la teoría del enojo estado-rasgo (Alcázar-Olán \& Deffenbaher, 2013b; Deffenbacher et al., 1996; Spielberger, 1999). Aunque la teoría se formuló originalmente para adultos, la hipótesis de agresión se cumplió también en adolescentes. Los jóvenes con alto enojo rasgo tuvieron más episodios de agresiones físicas que los jóvenes con bajo enojo. En consecuencia, la teoría del enojo estado rasgo fortalece su universalidad y su presencia en distintas etapas del desarrollo (adolescencia y adultez).

Las y los jóvenes fueron más similares que distintos en cuanto a la actualidad de su expresión iracunda. En general, varones y mujeres tuvieron episodios recientes donde mostraron su molestia agresivamente. Sólo se observaron 
diferencias en cuatro de doce variables. Los varones retaron a golpes y golpearon la pared, mientras que las mujeres aventaron cosas y azotaron la puerta. La varianza explicada atribuible al sexo fue mínima, de 1.7 a 3.7 \%. Estos resultados replican la relativa ausencia de diferencias entre sexos con respecto al enojo (Archer, 2004; Deffenbacher, Alcázar-Olán, Kocur, \& Richards, 2014). En momentos de enojo, varones y mujeres son más parecidos que diferentes.

Ante la falta de diferencias por sexo, cabe la posibilidad de que la expresión y el control del enojo estén más determinados por factores distintos a los roles de género. El enojo no parece depender del sexo, más bien, el enojo surge siempre que una persona experimente una situación incómoda, desagradable o aversiva (Berkowitz, 1996). No es necesario ser varón o mujer para sentirse incómodo. Tampoco es necesario ser varón o mujer para expresar (o no) el enojo ni para controlar (o no) dicha emoción.

Los resultados deben someterse a consideración con base en el marco de algunas limitaciones. Primero, aunque preguntamos a los jóvenes por la última ocasión en que expresaron su enojo de forma físico-agresiva, sus respuestas no son sinónimo de expresión frecuente del enojo. Es posible que una persona mostrara su enojo la semana pasada al golpear a alguien, asimismo, que dicho episodio sea tal vez el único, porque tal persona no volverá a pelear dentro de muchos meses o años. Estrictamente, la expresión del enojo con agresión física reciente no es equivalente a expresión frecuente del enojo. El estudio partió del supuesto de que el enojo rasgo es un patrón estable de respuesta (Spielberger, 1999). En consecuencia, cabe esperar que los individuos cuya expresión del enojo agresivo sucede de forma reciente, lo hacen y lo seguirán haciendo así (Baron \& Richardson, 1994).

Segundo, la agresión es un tema poco aceptado socialmente. Algunos jóvenes prefieren no hablarlo por pena o no evidenciar sus comportamientos. Como resultado, las respuestas a los cuestionarios pueden alterarse para no informar de manera precisa (Tourangeau, Rips, \& Rasinski, 2000). Suponemos que esta limitación se redujo porque los cuestionarios no pidieron los nombres de los alumnos y los profesores de la escuela no aplicaron aquellos ni estuvieron presentes durante el proceso. Estudios futuros pueden evaluar la deseabilidad social junto con la agresión para identificar si los jóvenes mienten al dar respuestas socialmente correctas.

Tercero, el método para recabar los datos fue el cuestionario, no la observación directa. El cuestionario provocó algunas inconsistencias en las respuestas con la opción de que el joven Nunca ha hecho la conducta agresiva. En particular, hay inconsistencias en las tablas 4 y 5 . No es posible que $9.6 \%$ de los jóvenes con alto enojo rasgo diga que nunca ha azotado la puerta (ver tabla 4) y luego $13.6 \%$ y 11.2 $\%$ de los mismos jóvenes dijeron que nunca lo han hecho (ver tabla 5). Estas inestabilidades en las respuestas (o en la captura) hicieron variar ligeramente los porcentajes en la opción de Nunca lo he hecho, de $1.4 \%$ a $4.7 \%$, por lo 
cual no consideramos que sea un defecto grave. Los números de las tablas deben tomarse como aproximaciones. Además, los cuestionarios son apropiados para investigar emociones como el enojo y son un método sin riesgo y sin daño para evaluar la agresión.

En síntesis, a diferencia de los adolescentes con bajo enojo rasgo, los de alto enojo rasgo: 1) mostraron su molestia con agresiones físicas recientes hacia otras personas (e. g., empujar a alguien) y hacia el ambiente (e. g., golpear la pared); 2) aventaron cosas contra sus hermanos/ as durante el último mes, la última semana e incluso hoy; y 3) azotaron la puerta estando enojados en la mañana, la tarde y la noche, cuando estuvieron en casa. Las relaciones entre hermanos y las casas de los adolescentes fungen como situaciones y escenarios donde predominó la expresión del enojo con agresión física.

\section{Referencias}

Alcázar-Olán, R. J., \& Deffenbacher, J. L. (2013a). High trait anger mexican youth: Characteristics, parental anger, and counseling needs. The Spanish Journal of Psychology, 16, 1-10. doi: 10.1017/sjp.2013.89

Alcázar-Olán, R. J., \& Deffenbacher, J. L. (2013b). State-trait anger theory and hypotheses. Rúbricas: Revista de la Universidad Iberoamericana Puebla, 5, 30-34.

Alcázar-Olán, R. J., Deffenbacher, J. L., PoolCibrián, W. J., Reyes-Pérez, V., \& Hernández-Guzmán, L. (2014). Developing a valid version of the ML-STAXI to measure anger in mexican adolescents. En M. G. Penrod
\& S. N. Paulk (Eds.), Psychology of Anger: New Research (pp. 89-105). New York: Nova publishers.

Archer, J. (2004). Sex differences in aggression in real-world settings: A meta-analytic review. Review of General Psychology, 8, 291322. doi: 10.1037/1089-2680.8.4.291

Averill, J. R. (1983). Studies on anger and aggression: Implications for theories of emotion. American Psychologist, 38, 11451160. doi: 10.1037/0003-066X.38.11.1145

Baron, R. A., \& Richardson, D. R. (1994). Human Aggression (2nd ed.). Nueva York: Plenum Press.

Berkowitz, L. (1996). Agresión: Causas, consecuencias, control. Bilbao, España: Desclée de Brouwer.

Blanchard-Fields, F., \& Coats, A. H. (2008). The experience of anger and sadness in everyday problems impacts age differences in emotion regulation. Developmental Psychology, 44, 1547-1556. doi: 10.1037/a0013915

Campano, J. P., \& Munakata, T. (2004). Anger and aggression among Filipino students. Adolescence, 39(156), 757-764.

Cautin, R., Overholser, J. C., \& Goetz, P. (2001). Assessment of mode of anger expression in adolescent psychiatric inpatients. Adolescence, 36(141), 163-170.

Cohen, J. (1988). Statistical power analysis for the behavioral sciences (2nd ed.). Hillsdale, NJ: Erlbaum.

Deffenbacher, J. L., Alcázar-Olán, R. J., Kocur, J. L., \& Richards, T. L. (2014). A test of the state-trait model of anger with middle-aged 
drivers. En M. G. Penrod \& S. N. Paulk (Eds.), Psychology of anger: New research (pp. 71-88). New York: Nova publishers.

Deffenbacher, J. L., Oetting, E. R., Thwaites, G. A., Lynch, R. S., Baker, D. A., Stark, R. S... Eiswerth-Cox, L. (1996). State-trait anger theory and the utility of the trait anger scale. Journal of Counseling Psychology, 43, 131148. doi: 10.1037/0022-0167.43.2.131

Hubbard, J. A., Romano, L. J., McAuliffe, M. D., \& Morrow, M. T. (2010). Anger and reactive-proactive aggression distinction in childhood and adolescence. En. M. Potegal, G. Stemmler \& C. Spielberger (Eds.), International handbook of anger (pp. 231-239). NewYork: Springer.

Jackson, J., Kuppens, P., Sheeber, L. B., \& Allen, N. B. (2011). Expression of anger in depressed adolescents: The role of the family environment. Journal of Abnormal and Child Psychology, 39, 463-474.

Lee, J., Choi, H., Kim, M. J., Park, C. G., \& Shin, D. (2009). Anger as a predictor of suicidal ideation in middle-school students in Korea: Gender differences in threshold point. Adolescence, 44(174), 433-446.

Loeber, R., \& Hay, D. (1997). Key issues in the development of aggression and violence from childhood to early adulthood. Annual Review of Psychology, 48, 371-410.

Moscoso, M. S., \& Spielberger, C. D. (1999). Evaluación de la experiencia, expresión y control de la cólera en Latinoamérica. Revista Psicología Contemporánea, 6(6), 4-13.
Peled, M., \& Moretti, M. M. (2007). Rumination on anger and sadness in adolescence: Fueling of fury and deepening of despair. Journal of Clinical and Adolescent Psychology, 36(1), 66-75.

Quinn, C. A., Rollock, D., \& Vrana, S. R. (2014). A test of Spielberger's state-trait theory of anger with adolescents: Five hypotheses. Emotion, 14(1), 74-84. doi: 10.1037/a0034031

Schieman, S. (2010). The sociological study of anger: Basic social patterns and contexts. En M. Potegal, G. Stemmler \& C. Spielberger (Eds.), International handbook of anger (pp. 329-347). NewYork: Springer.

Schwarz, N. (1999). Self-reports: How the questions shape the answers. American Psychologist, 54, 93-105.

Spielberger, C. D. (1999). Manual for the StateTrait Anger Expression Inventory-Revised. Odessa, FL: Psychological Assessment Resources.

Tourangeau, R., Rips, L. J., \& Rasinski, K. (2000). The psychology of survey response. New York: Cambridge University Press.

Winkielman, P., Knäuper, B., \& Schwarz, N. (1998). Looking back at anger: Reference periods change the interpretation of emotion frequency questions. Journal of Personality and Social Psychology, 75, 719-728. 